\title{
Mappings of finite distortion: Sharp Orlicz-conditions
}

\author{
Janne Kauhanen, Pekka Koskela, Jan Malý, \\ Jani Onninen and Xiao Zhong
}

\begin{abstract}
We establish continuity, openness and discreteness, and the condition $(N)$ for mappings of finite distortion under minimal integrability assumptions on the distortion.
\end{abstract}

\section{Introduction}

This paper is part of our program to establish the fundamentals of the theory of mappings of finite distortion [5], [1], [6], [9], [10] which form a natural generalization of the class of quasiregular mappings, also called mappings of bounded distortion. In the previous papers we considered mappings $f \in W^{1,1}\left(\Omega, \mathbb{R}^{n}\right)$ of exponentially integrable distortion. Here and throughout the paper, $\Omega \subset \mathbb{R}^{n}$ is an open, connected set. If $f \in W^{1,1}\left(\Omega, \mathbb{R}^{n}\right)$ satisfies

$$
|D f(x)|^{n} \leq K(x) J(x, f) \quad \text { a.e., }
$$

where $K(x)<\infty$ and if $J(\cdot, f) \in L_{\text {loc }}^{1}(\Omega)$, we say that $f$ is a mapping of finite distortion. We call $f$ a mapping of exponentially integrable distortion if furthermore $\exp (\lambda K) \in L_{\text {loc }}^{1}(\Omega)$ for some $\lambda>0$. Mappings of exponentially integrable distortion in this sense were shown to have many of the nice properties of a mapping of bounded distortion. Regarding the necessity of the exponential integrability, an example from [9] shows that no topological properties like openness can be expected if we merely assume that $\exp \left(K / \log (e+K)^{2}\right)$ be integrable. In this paper we further examine the integrability assumptions on $K$. Let us replace the assumption $\exp (\lambda K) \in L_{\text {loc }}^{1}(\Omega)$ with $\exp (\Psi(K)) \in L_{\text {loc }}^{1}(\Omega)$. By the above, the critical 
power-like behavior of $\Psi$ is linear. For the first theorem, we assume that $\Psi$ is a strictly increasing, differentiable function, and we make the following two assumptions, the second of which is entirely harmless (see Remark 2.2):

$(\Psi-1) \quad \int_{1}^{\infty} \frac{\Psi^{\prime}(t)}{t} d t=\infty$

$(\Psi-2) \lim _{t \rightarrow \infty} t \Psi^{\prime}(t)=\infty$.

Then we have the following regularity result.

Theorem 1.1. Suppose that $\Psi$ satisfies $(\Psi-1)$ and $(\Psi-2)$. Let $f$ be a mapping of finite distortion $K$ with $\exp (\Psi(K)) \in L_{\text {loc }}^{1}(\Omega)$ and suppose that $\operatorname{det} D f=$ $J(\cdot, f) \in L_{\text {loc }}^{1}(\Omega)$. Then $f$ is continuous and either constant or both open and discrete. Moreover, $f$ maps sets of Lebesgue measure zero to sets of measure zero.

The continuity here means the existence of a continuous representative. The claims of Theorem 1.1 were established in [6], [9] and [10] for $\Psi(t)=\lambda t, \lambda>0$. In the planar setting, Theorem 1.1 is partially covered by the results in [7].

As practical examples, Theorem 1.1 allows for

$$
\Psi(t)=t, \frac{t}{\log (e+t)}, \frac{t}{\log (1+t) \log \log \left(e^{e}+t\right)}, \ldots
$$

for any string of iterated logarithms. Regarding the sharpness, we will show, in particular, that

$$
\Psi(t)=\frac{t}{t^{\epsilon}}, \frac{t}{\log ^{1+\epsilon}(e+t)}, \frac{t}{\log (e+t) \log ^{1+\epsilon} \log \left(e^{e}+t\right)}, \ldots
$$

are not sufficient, for any $\epsilon>0$. This easily follows from our next result that is a substantial improvement on the construction that we gave in [9], also see [7] regarding the part (a).

Theorem 1.2. Suppose that $\Psi$ is a strictly increasing function and

$$
\int_{1}^{\infty} \frac{\Psi^{\prime}(s)}{s} d s<\infty .
$$

(a) There exists a mapping $f: \mathbb{B} \rightarrow \mathbb{R}^{n}$ of finite distortion $K(x)=\frac{|D f(x)|^{n}}{J(x, f)}$, with integrable Jacobian, with

$$
\int_{\mathbb{B}} \exp [\Psi(K(x))] d x<\infty
$$

and so that $f$ maps $\mathbb{B} \backslash\{0\}$ homeomorphically onto the annulus $\{x \in$ $\left.\mathbb{R}^{n}: 1<|x|<b\right\}$. In particular, $f$ has no continuous representative. 
(b) There exists a continuous, non-constant mapping $f: Q_{0}=[0,1]^{n} \rightarrow \mathbb{R}^{n}$ of finite distortion $K(x)=\frac{|D f(x)|^{n}}{J(x, f)}$, with integrable Jacobian, with

$$
\int_{Q_{0}} \exp [\Psi(K(x))] d x<\infty
$$

and so that $f$ is neither open, nor discrete, and it maps a set of measure zero to a set of positive measure.

Theorem 1.1 is based on the arguments in [6], [9], [10] together with the following new observations. The integrability conditions on $K, \Psi$ guarantee that $\Phi(|D f|)$ is locally integrable in $\Omega$ for a strictly increasing function $\Phi$ that satisfies the conditions

$(\Phi-1) \int_{1}^{\infty} \frac{\Phi(t)}{t^{1+n}} d t=\infty$.

$(\Phi-2)$ There is $p \in(n-1, n)$ such that $t \mapsto t^{-p} \Phi(t)$ increases for large values of $t$.

Secondly, relying on recent results in [4], [11] and [3], we conclude that the point-wise Jacobian $J(x, f)$ then coincides with the so-called distributional Jacobian. This is the key fact in many of the estimates in [6], [9], [10] and we obtain the proposed topological and analytical results.

In the course of this argument we in fact establish the following result.

Theorem 1.3. Let $f \in W_{\text {loc }}^{1,1}\left(\Omega, \mathbb{R}^{n}\right)$ be a mapping of finite distortion $K$. Suppose that $\Phi(|D f|)+K^{q} \in L_{\text {loc }}^{1}(\Omega)$, with $q>n-1$ and $\Phi$ satisfying $(\Phi-1)$ and $(\Phi-2)$. Then $f$ is continuous and either constant or both open and discrete. Moreover, $f$ maps sets of Lebesgue measure zero to sets of measure zero.

Here, the assumption $(\Phi-1)$ is critical: the examples referred to in part (b) of Theorem 1.2 satisfy

$$
\Phi(|D f|)+\exp [\Psi(K(x))] \in L^{1}\left(Q_{0}\right)
$$

see formulas (3.10) and (3.13). The assumption $(\Phi-2)$ is also necessary. For (a), it is enough to consider $f(x)=x(1+|x|) /|x|$, then $\Phi(D f) \in L^{1}(\mathbb{B})$ for any $\Phi$ violating $(\Phi-2)$. Concerning the necessity for (b), see Remark 3.1. Thus Theorem 1.3 gives a sharp extension of the celebrated results by Reshetnyak (c.f. [13], [14], [15]) on mappings of bounded distortion. It still remains unknown if the $L^{n-1}$-integrability of $K$ is already sufficient under the given assumptions on $|D f|$; this is not known even when $|D f| \in L^{n}(\Omega)$. For this see [12], the monograph [8], and the references therein. 


\section{Proof of Theorem 1.1}

We call a continuously differentiable and strictly increasing function $\Psi$ : $[0, \infty) \rightarrow[0, \infty)$ with $\Psi(0)=0$ and $\lim _{t \rightarrow \infty} \Psi(t)=\infty$ an Orlicz function.

In the course of this section, we associate with $\Psi$ two other Orlicz functions (see equation $(2.2)$ ):

$$
\begin{aligned}
& \psi(t)=t \exp (\Psi(t)), \\
& g(s)=\frac{s}{\psi^{-1}(s)}-1, s>0, \text { and } g(0)=0 .
\end{aligned}
$$

We notice that $\psi$ is strictly increasing so that the inverse function $\psi^{-1}$ makes sense. We immediately have

$$
g(\psi(t))=\exp (\Psi(t))-1 .
$$

In the first lemma we do not assume $(\Psi-2)$.

Lemma 2.1. Assume that $\Psi$ is an Orlicz function satisfying $(\Psi-1)$. Then

(a) $\int_{1}^{\infty} \frac{g(s)}{s^{2}} d s=\infty$ and

(b) given $a, b \geq 0$ we have

$$
g(a b) \leq a+\exp (\Psi(b))-1 .
$$

Proof. By the change of variables $s=\psi(t)$ and (2.2) we obtain

$$
\begin{aligned}
\int_{\psi(1)}^{\infty} \frac{g(s)+1}{s^{2}} d s & =\int_{1}^{\infty} \frac{(g(\psi(t))+1) \psi^{\prime}(t)}{\psi(t)^{2}} d s \\
& =\int_{1}^{\infty} \frac{\psi^{\prime}(t)}{t \psi(t)} d t \\
& =\int_{1}^{\infty} \frac{\left(1+t \Psi^{\prime}(t)\right) \exp (\Psi(t))}{t^{2} \exp (\Psi(t))} d t \\
& =\int_{1}^{\infty}\left(\frac{1}{t^{2}}+\frac{\Psi^{\prime}(t)}{t}\right) d t=\infty .
\end{aligned}
$$

This proves (a). Regarding (b), we distinguish two cases; naturally we may assume that $a \neq 0 \neq b$. If $a b \leq \psi(b)$, then by $(2.2)$

$$
g(a b) \leq g(\psi(b))=\exp (\Psi(b))-1 .
$$

If $a b \geq \psi(b)$, then

$$
g(a b)=\frac{a b}{\psi^{-1}(a b)}-1 \leq \frac{a b}{b}-1=a-1 .
$$


Remark 2.2. The condition $(\Psi-1)$ is crucial for our considerations and shown to be necessary by our counterexamples. However, this condition alone is too weak for our purposes. To demonstrate this, let us consider a sequence $\left\{a_{k}\right\}$ with $a_{k+1}>k a_{k}$ and function $\Psi$ which increases from $2 a_{k-1}$ to $a_{k}$ on $\left[a_{k}, 2 a_{k}\right]$ and from $a_{k}$ to $2 a_{k}$ on $\left[2 a_{k}, a_{k+1}\right]$. Then

$$
\int_{a_{k}}^{2 a_{k}} \frac{\Psi^{\prime}(t)}{t} d t \geq \frac{a_{k}-2 a_{k-1}}{2 a_{k}} \longrightarrow \frac{1}{2}
$$

and thus $(\Psi-1)$ is verified. On the other hand, if $e^{2 a_{k}}<a_{k+1}^{1 / k}$ then $\exp (\Psi(t))$ is not comparable with any $t^{q}, q>1$. This means also that integrability of $\exp (\Psi(K))$ would not imply integrability of $K^{q}$.

This consideration shows that something should be added to the condition $(\Psi-1)$. The condition $(\Psi-1)$ implies that $\lim \sup _{t \rightarrow \infty} t \Psi^{\prime}(t)=\infty$. It will not exclude important examples of Orlicz functions if we assume that this limsup turns to limit. Among power-like functions $\Psi(t)=t^{\alpha},(\Psi-1)$ corresponds to $\alpha<1$, while $(\Psi-2)$ is true for all $\alpha>0$. This explains in what sense we regard $(\Psi-2)$ to be "harmless".

Lemma 2.3. Assume that $\Psi$ is an Orlicz function satisfying $(\Psi-2)$ and $\varepsilon \in$ $(0,1)$. Then there exists $s_{0} \in(0, \infty)$ such that the functions $h: s \mapsto s^{\varepsilon-1} g(s)$ is increasing on $\left(s_{0}, \infty\right)$.

Proof. By (2.2) we rewrite

$$
h(\psi(t))=\psi(t)^{\varepsilon-1}\left(1+g(\psi(t))=t^{\varepsilon-1} \exp (\varepsilon \Psi(t)) .\right.
$$

Hence

$$
(h(\psi(t)))^{\prime}=t^{\varepsilon-2} \exp (\varepsilon \Psi(t))\left[\varepsilon t \Psi^{\prime}(t)-(1-\varepsilon)\right] .
$$

By $(\Psi-2)$ we find a $t_{0}$ such that $h(\psi(t))$ increases for $t>t_{0}$. We conclude that $h(s)=s^{\varepsilon-1}(g(s)+1)-s^{\varepsilon-1}$ is increasing on $\left(s_{0}, \infty\right)$, where $s_{0}=\psi\left(t_{0}\right)$.

Now we collect results which enable us to derive regularity properties of a mapping of finite distortion from integrability of its differential. Let us consider a class $X(\Omega) \subset L^{n-1}(\Omega)$ of measurable functions on $\Omega$ satisfying the following two conditions:

$(\mathrm{X}-1) J(\cdot, f) \in L_{\mathrm{loc}}^{1}(\Omega)$ and $\operatorname{det} D f=\operatorname{Det} D f$ provided $f \in W^{1,1}\left(\Omega, \mathbb{R}^{n}\right)$, $|D f| \in X(\Omega)$ and $J(\cdot, f) \geq 0$ a.e.

(X-2) if $g, h \geq 0$ are measurable, $g \leq c h$ for some $0<c<\infty$ and $h \in X(\Omega)$, then $g \in X(\Omega)$. 
Here the statement $\operatorname{det} D f=\operatorname{Det} D f$ means that

$$
\int_{\Omega} \varphi J(x, f) d x=-\int_{\Omega} f_{i} J\left(x, f_{1}, \ldots, f_{i-1}, \varphi, f_{i+1}, \ldots, f_{n}\right) d x
$$

for each $i=1, \ldots, n$ and for all $\varphi \in C_{0}^{\infty}(\Omega)$.

The following proposition states the weak monotonicity of a mapping $f$, see [6, Definition 1.5], under assumptions which are adapted to our situation.

Proposition 2.4. Let $X$ be a space of measurable functions satisfying (X-1) and (X-2). Let $f=\left(f_{1}, \ldots, f_{n}\right) \in W^{1, n-1}(\Omega)$ be a mapping of finite distortion with $|D f| \in X(\Omega)$. Then the coordinate functions of $f$ are weakly monotone.

Proof. We follow the standard idea as in [6, Section 4]. Let us consider a ball $B \subset \subset \Omega$. We prove e.g. that if $f_{1} \leq M$ on $\partial B$ in the sense of traces, i.e. the positive part of $f_{1}-M$ belongs to $W_{0}^{1,1}(B)$, then $f_{1} \leq M$ a.e. in $B$. We consider the truncated function $\tilde{f}_{1}=\min \left(f_{1}, M\right)$ and the mapping $\tilde{f}=\left(\tilde{f}_{1}, f_{2}, \ldots, f_{n}\right)$. Notice that, by $(\mathrm{X}-2),|D \tilde{f}| \in X(\Omega)$. Let $\varphi$ be a smooth test function with compact support in $\Omega$ such that $\varphi=1$ on $B$. Since $f_{1}$ differs from $\tilde{f}_{1}$ only on $B$ where $D \varphi=0$, we have $f_{1} D \varphi=\tilde{f}_{1} D \varphi$, and thus

$$
\begin{aligned}
\int_{\Omega} \varphi J(x, f) d x & =-\int_{\Omega} f_{1} J\left(x, \varphi, f_{2}, \ldots, f_{n}\right) d x \\
& =-\int_{\Omega} \tilde{f}_{1} J\left(x, \varphi, f_{2}, \ldots, f_{n}\right) d x=\int_{\Omega} \varphi J(x, \tilde{f}) d x
\end{aligned}
$$

Hence, if we set $E=\{\tilde{f} \neq f\}$, we have

$$
\int_{E} J(x, f) d x=\int_{E} J(x, \tilde{f}) d x=0 .
$$

Since $J(x, f) \geq 0$, it follows that $J f=0$ a.e. on $E$ and thus, as $f$ is a mapping of finite distortion, $D f=0$ a.e. in $E$. It follows that $D\left(f_{1}-\tilde{f}_{1}\right)=0$ a.e. in $\Omega$ which yields that $f_{1}=\tilde{f}_{1} \leq M$ a.e. in $B$.

The following proposition summarizes the outcome of [9] and [10].

Proposition 2.5. Let $X$ be a space of measurable functions satisfying (X-1) and $(\mathrm{X}-2)$. Let $f=\left(f_{1}, \ldots, f_{n}\right) \in W^{1, n-1}(\Omega)$ be a mapping of finite distortion $K \in L^{q}(\Omega), q>n-1$, and $|D f| \in X(\Omega)$. Suppose that $f$ is continuous. Then $f$ is open and discrete and maps sets of measure zero to sets of measure zero. 
Proof. In [9, Theorems 2.1, 2.4, 3.1] it was shown that a mapping satisfying the hypotheses is open, discrete, and sense-preserving. By [10, Lemma 3.2], a continuous sense-preserving mapping $f \in W^{1, p}\left(\Omega, \mathbb{R}^{n}\right), p>n-1$, for which det $D f=\operatorname{Det} D f$ maps sets of measure zero to sets of measure zero. We only need to check that $f \in W^{1, p}\left(\Omega, \mathbb{R}^{n}\right)$ for some $p>n-1$. Because of the locality of our claim, it suffices to check that $|D f| \in L_{\mathrm{loc}}^{p}(\Omega)$ for some $p>$ $n-1$, which follows by means of the Hölder inequality from the assumption $J(\cdot, f) \in L_{\mathrm{loc}}^{1}(\Omega)$ and from the fact that $K \in L_{\mathrm{loc}}^{q}(\Omega)$ with $q>n-1$.

The assumption $\int_{\Omega} \Phi(|D f(x)|) d x<\infty$ with $\Phi$ as above has two important consequences.

Proposition 2.6. [11, Corollary 1.3] Let $\Phi$ be an Orlicz-function that satisfies $(\Phi-1)$ and $(\Phi-2)$. Let $f \in W^{1,1}\left(\Omega, \mathbb{R}^{n}\right)$ satisfy $J(x, f) \geq 0$ a.e. $x \in \Omega$, and assume that $\int_{\Omega} \Phi(|D f(x)|) d x<\infty$. Then $\operatorname{det} D f \in L_{\mathrm{loc}}^{1}(\Omega)$ and $\operatorname{det} D f=\operatorname{Det} D f$.

The following proposition is essentially [6, Theorem 1.6], but with slightly weakened assumptions on $\Phi$.

Proposition 2.7. Let $\Phi$ be an Orlicz-function that satisfies $(\Phi-1)$ and $(\Phi-2)$. Let $u \in W^{1,1}(\Omega)$ be a weakly monotone function, and assume that $\int_{\Omega} \Phi(|D u(x)|) d x<\infty$. Then u has a continuous representative.

Proof. We will follow the proof of [6, Theorem 1.6] with a small modification. By $C$ we denote various constants which may change from line to line. Fix a point $a \in \Omega$ and $R>0$ with $B(a, 2 R) \subset \Omega$, and denote by $\omega(r)$ the essential oscillation of $u$ on $B(a, r), 0<r<R$. By [6, Lemma 7.2], for almost every radius $r \in(0, R)$ we have

$$
\omega(r)^{p} \leq C r^{p-n+1} \int_{\partial B(a, r)}|\nabla u|^{p} d S .
$$

We consider a $t_{0}$ such that $t^{-p} \Phi(t)$ is increasing on $\left(t_{0}, \infty\right)$ and a constant $\tau$ such that

$$
\Phi(\tau)=f_{\partial B(a, r)} \Phi(\nabla u) d S,
$$

where $f$ stands for the integral average. Write $\lambda=\max \left(\tau, t_{0}\right)$. Then we estimate

$$
\begin{aligned}
\int_{\partial B(a, r)}|\nabla u|^{p} d S & \leq \int_{\partial B(a, r) \cap\{|\nabla u|>\lambda\}}|\nabla u|^{p} d S+\int_{\partial B(a, r) \cap\{|\nabla u| \leq \lambda\}}|\nabla u|^{p} d S \\
& \leq \frac{\lambda^{p}}{\Phi(\lambda)} \int_{\partial B(a, r)} \Phi(\nabla u) d S+C r^{n-1} \lambda^{p} \leq 2 C r^{n-1} \lambda^{p} .
\end{aligned}
$$


It follows that

$$
\frac{\omega(r)}{C r} \leq \lambda
$$

and thus

$$
\Phi\left(\frac{\omega(r)}{C r}\right) \leq \Phi(\lambda) \leq f_{\partial B(a, r)}\left[\Phi\left(t_{0}\right)+\Phi(\nabla u)\right] d S .
$$

Now we may continue as in the proof of [6, Theorem 1.6].

Proof of Theorem 1.3. Proposition 2.6 shows that our space $L^{\Phi}(\Omega)$ qualifies for $X(\Omega)$ with $(\mathrm{X}-1)$ and $(\mathrm{X}-2)$. By Proposition 2.4 we see that the coordinate functions of $f$ are weakly monotone which implies continuity by Proposition 2.7. Then Proposition 2.5 yields the conclusion.

Proof of Theorem 1.1. Let $\Phi(t)=g\left(t^{n}\right)$ where $g$ is as in (2.1). Then by Lemma 2.1 (b)

$$
\begin{aligned}
\int_{\Omega} \Phi(|D f|) d x & =\int_{\Omega} g\left(|D f|^{n}\right) d x \leq \int_{\Omega} g(J(x, f) K(x)) d x \\
& \leq \int_{\Omega} J(x, f) d x+\int_{\Omega} \exp (K(x)) d x<\infty .
\end{aligned}
$$

By Lemma 2.3 and Lemma 2.1 (a), the function $\Phi$ satisfies $(\Phi-1)$ and $(\Phi-2)$ (for all $p \in(n-1, n)$ ), and the inclusion $L_{\text {loc }}^{\Phi}(\Omega) \subset L_{\text {loc }}^{p}(\Omega)$ holds for all $p \in(n-1, n)$. Hence the assumptions of Theorem 1.3 are verified.

\section{Proof of Theorem 1.2}

We begin by giving examples of discontinuous mappings of finite distortion with the distortion function having the desired degree of regularity (also see [7]). We consider mappings $f: \mathbb{B} \rightarrow \mathbb{R}^{n}$ of the form

$$
f(x)=\frac{x}{|x|} \rho(|x|) .
$$

The function $t \rightarrow \rho(t)$, for $0 \leq t \leq 1$, will continuously increase from the value 1 at $t=0$ to $b>1$ at $t=1$. Thus $f$ will map homeomorphically the punctured unit ball $\mathbb{B} \backslash\{0\}$ onto the annulus $\left\{x \in \mathbb{R}^{n}: 1<|x|<b\right\}$. We may calculate the differential matrix of $f$ and its determinant by using the familiar formulas

$$
D f(x)=\frac{\rho(|x|)}{|x|} \mathbf{I}+\left(\rho^{\prime}(|x|)-\frac{\rho(|x|)}{|x|}\right) \frac{x \otimes x}{|x|^{2}},
$$


where $x \otimes x$ is the $n \times n$ matrix whose $i, j$-entry equals $x_{i} x_{j}$, and

$$
J(x, f)=\rho^{\prime}(|x|)\left(\frac{\rho(|x|)}{|x|}\right)^{n-1} .
$$

Our choice for $\rho$ will satisfy

$$
\rho^{\prime}(t) \leq \beta \frac{\rho(t)}{t}
$$

for some $\beta \geq 1$. Consequently, the norm of differential matrix in question satisfies

$$
|D f(x)| \leq(\beta+2) \frac{\rho(|x|)}{|x|}
$$

and the dilatation function $\mathrm{K}$ satisfies

$$
K(x)=\frac{|D f(x)|^{n}}{J(x, f)} \leq(\beta+2)^{n} \frac{\rho(|x|)}{|x| \rho^{\prime}(|x|)} .
$$

We may assume that $\Psi(1)=1$. We define $\rho$ by setting

$$
\rho(t)=\exp \left(\lambda \int_{\Psi^{-1}\left(\log \frac{e}{t}\right)}^{\infty} \frac{\Psi^{\prime}(s)}{s} d s\right)
$$

for $0<t<1$, where $\lambda$ is a constant, whose value will be determined later. Using the change of variables

$$
s=\Psi^{-1}\left(\log \frac{e}{r}\right)
$$

we obtain

$$
\rho(t)=\exp \left(\lambda \int_{0}^{t} \frac{d r}{r \Psi^{-1}\left(\log \frac{e}{r}\right)}\right) .
$$

For the Jacobian integral we compute

$$
\int_{\mathbb{B}} J(x, f) d x=C(n) \int_{0}^{1} \rho^{n-1}(t) \rho^{\prime}(t) d t=C(n)\left(\rho^{n}(1)-\rho^{n}(0)\right)<\infty .
$$

We also have

$$
\frac{t \rho^{\prime}(t)}{\rho(t)}=t(\log \rho(t))^{\prime}=\frac{\lambda}{\Psi^{-1}\left(\log \frac{e}{t}\right)} .
$$


This quantity tends to zero as $t \rightarrow 0$ and thus there exists $t_{0}>0$ such that (3.4) follows with constant 1 for all $t \in\left(0, t_{0}\right)$. Fix $\lambda=3^{n}$. By (3.6) and (3.7) we obtain

$$
\begin{aligned}
\exp \Psi(K(x)) & \leq \exp \Psi\left(\frac{3^{n} \rho(|x|)}{|x| \rho^{\prime}(|x|)}\right) \\
& \leq \exp \Psi\left(\Psi^{-1}\left(\log \frac{e}{|x|}\right)\right)=\frac{e}{|x|}
\end{aligned}
$$

for all $x \in B\left(0, t_{0}\right) \backslash\{0\}$. Hence $\exp \Psi \circ K \in L^{1}(\mathbb{B})$, as desired.

The construction we need for part (b) of Theorem 1.2 is a substantial improvement on the construction in [9]. For the convenience of the reader we present here also the part of the construction from [9] that need not be altered.

We begin by introducing some notation. Besides the usual Euclidean norm $|x|=\left(x_{1}^{2}+\cdots+x_{n}^{2}\right)^{1 / 2}$ we will use the cubic norm $\|x\|=\max _{i}\left|x_{i}\right|$. Using the cubic norm, the $x_{0}$-centered closed cube with edge length $2 r>0$ and sides parallel to coordinate axes can be represented in the form

$$
Q\left(x_{0}, r\right)=\left\{x \in \mathbb{R}^{n}:\left\|x-x_{0}\right\| \leq r\right\}
$$

We then call $r$ the radius of $Q$. Let us denote $c Q\left(x_{0}, r\right)=Q\left(x_{0}, c r\right)$ if $c>0$. We will use the notation $a \lesssim b$ if there is a constant $c>0$ (not depending on (integration) variables or summation indices) such that $a \leq c b$, and we write $a \approx b$ if $a \lesssim b$ and $b \lesssim a$. For technical reasons we will assume that $\Psi(1)=1$.

We will prove part (b) of Theorem 1.2 by giving a mapping $f: Q_{0} \rightarrow$ $\mathbb{R}^{n}$ so that $f=\operatorname{Id}$ on $\partial Q_{0}, J(x, f)<0$ a.e. and so that the rest of the requirements hold for $|J(x, f)|$; the desired mapping is then obtained by employing an auxiliary reflection in a hyperplane.

In the following, we will construct a sequence of continuous, piecewise continuously differentiable mappings $f_{k}: Q_{0} \rightarrow \mathbb{R}^{n}$. First we introduce a sequence of compact sets in $Q_{0}$ whose intersection is a Cantor set.

The unit cube $Q_{0}$ is first divided into $2^{n}$ cubes with radius $1 / 4$, which are each in turn divided into a subcube with radius $(1 / 4) / 2$ and a difference of two cubes which we refer to as an annulus. The family $\mathcal{Q}_{1}$ consists of these $2^{n}$ subcubes. The remainder of the construction is then self-similar. The subcube is divided into $2^{n}$ cubes which are each in turn divided into a subcube with radius $4^{-2} / 2$ and an annulus. The family $\mathcal{Q}_{2}$ consists of these $2^{2 n}$ subcubes (see Figure 1). Continuing this way, we get the families $\mathcal{Q}_{k}, k=1,2,3, \ldots$, for which the radius of $Q \in \mathcal{Q}_{k}$ is $r(Q)=r_{k}=2^{-2 k-1}$ and the number of cubes in $\mathcal{Q}_{k}$ is $\# \mathcal{Q}_{k}=2^{n k}$. It easily follows that the resulting Cantor set is of measure zero. 

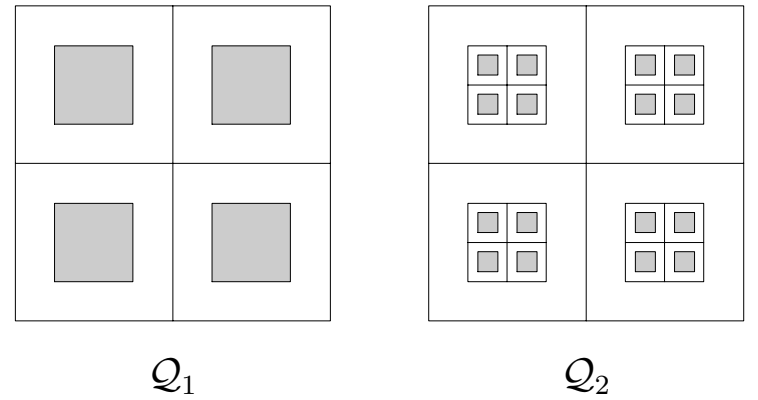

Figure 1: Families $\mathcal{Q}_{1}$ and $\mathcal{Q}_{2}$.

We are now ready to define the mappings $f_{k}$. Define $f_{0}(x)=x$. We will give a mapping $f_{1}$ that leaves the boundaries $\partial(2 Q), Q \in \mathcal{Q}_{1}$ fixed, turns each annulus $2 Q \backslash Q$ inside out and stretches the cube $Q$ so that $f_{1}$ is continuous (see Figure 2). The Jacobian determinant $J_{f_{1}}$ will be negative in each annulus $2 Q \backslash Q$ and positive in each cube $Q$. Next, $f_{2}$ equals $f_{1}$ in the annulae $2 Q \backslash Q, Q \in \mathcal{Q}_{1}$, turns each annulus $2 Q \backslash Q, Q \in \mathcal{Q}_{2}$, inside out, stretches the cube $Q$ and shifts the image so that $f_{2}$ is continuous. Moreover, $J_{f_{2}}$ is negative a.e. in $Q_{0} \backslash \bigcup_{Q \in \mathcal{Q}_{2}} Q$ and positive in $\bigcup_{Q \in \mathcal{Q}_{2}} Q$. We will then continue in this manner.

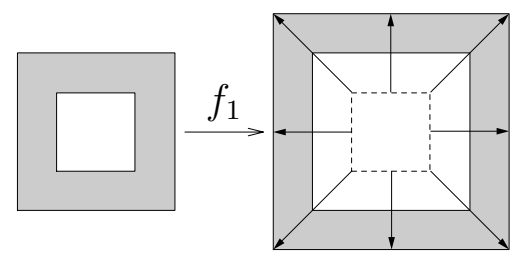

Figure 2: The mapping $f_{1}$ acting on $2 Q, Q \in \mathcal{Q}_{1}$.

To be precise, let $f_{0}(x)=x$ on $Q_{0}$ and let a sequence $\left\{\epsilon_{k}\right\}_{k \in \mathbb{N}}$ of small positive real numbers satisfy

$$
\sum_{k=1}^{\infty} \epsilon_{k}<\infty
$$

This sequence will be fixed later. For $k=1,2, \ldots$ define

$$
\varphi_{k}(r)= \begin{cases}2^{-k-1}\left(1+\epsilon_{1}\right) \cdots\left(1+\epsilon_{k-1}\right)\left(1+\frac{2 r_{k}-r}{r_{k}} \epsilon_{k}\right), & r_{k} \leq r \leq 2 r_{k} \\ 2^{-k-1}\left(1+\epsilon_{1}\right) \cdots\left(1+\epsilon_{k}\right) \frac{r}{r_{k}}, & 0 \leq r \leq r_{k}\end{cases}
$$


and

$$
f_{k}(x)= \begin{cases}f_{k-1}(x), & x \notin \bigcup_{Q \in \mathcal{Q}_{k}} 2 Q \\ f_{k-1}(z(Q))+\frac{x-z(Q)}{\|x-z(Q)\|} \varphi_{k}(\|x-z(Q)\|), & x \in 2 Q, Q \in \mathcal{Q}_{k} .\end{cases}
$$

Here $z(Q)$ is the center of the cube $Q$. Now

$$
\log \prod_{j=1}^{k}\left(1+\epsilon_{j}\right)=\sum_{j=1}^{k} \log \left(1+\epsilon_{j}\right) \leq \sum_{j=1}^{k} \epsilon_{j}
$$

and using the fact (3.8) we infer that

$$
\prod_{j=1}^{\infty}\left(1+\epsilon_{j}\right)<\infty
$$

Thus

$$
\prod_{j=1}^{k}\left(1+\epsilon_{j}\right) \approx 1, \quad k=1,2, \ldots
$$

Using this we obtain

$$
\left|f_{k+1}(x)-f_{k}(x)\right| \lesssim 2^{-k}
$$

and so the sum

$$
\sum_{k=1}^{\infty}\left|f_{k+1}(x)-f_{k}(x)\right|
$$

and the sequence $\left(f_{k}\right)$ converge uniformly. Hence the limit $f=\lim _{k \rightarrow \infty} f_{k}$ is continuous. Clearly $f$ is differentiable almost everywhere, its Jacobian determinant is strictly negative almost everywhere, and $f$ is absolutely continuous on almost all lines parallel to coordinate axes.

We next estimate $|D f(x)|,|J(x, f)|$ and $K(x)$ at $x \in \operatorname{int}(2 Q \backslash Q)$, $Q \in \mathcal{Q}_{k}$. Fix $k \in \mathbb{N}$. We see that, in the annulus $\operatorname{int}(2 Q \backslash Q), f$ is a radial mapping: $f(x)=(x /\|x\|) \varphi_{k}(\|x\|)$. Hence we have

$$
|D f(x)| / C_{1}(n) \leq \max \left\{\frac{\varphi_{k}(\|x\|)}{\|x\|},\left|\varphi_{k}^{\prime}(\|x\|)\right|\right\} \leq C_{1}(n)|D f(x)|
$$

and

$$
J_{f}(x) / C_{2}(n) \leq \frac{\varphi_{k}^{\prime}(\|x\|) \varphi_{k}(\|x\|)^{n-1}}{\|x\|^{n-1}} \leq C_{2}(n) J_{f}(x)
$$


a.e. in $\operatorname{int}(2 Q \backslash Q)$, see the formulas (3.2) and (3.3). By (3.8) and (3.9) we obtain

$$
|D f(x)| \lesssim \frac{\varphi_{k}\left(r_{k}\right)}{r_{k}} \approx 2^{k}
$$

and

$$
J(x, f) \approx\left(\frac{\varphi_{k}\left(r_{k}\right)}{r_{k}}\right)^{n-1} \varphi_{k}^{\prime}\left(r_{k}\right) \approx-\left(2^{k}\right)^{n-1} 2^{k} \epsilon_{k}=-2^{k n} \epsilon_{k}
$$

and finally

$$
K(x):=\frac{|D F(x)|}{|J(x, f)|} \lesssim \frac{2^{k n}}{2^{k n} \epsilon_{k}}=\frac{1}{\epsilon_{k}} .
$$

Since

$$
\left|\bigcup_{Q \in \mathcal{Q}_{k}} 2 Q \backslash Q\right| \approx 2^{-k n}
$$

we obtain in view of $(3.8)$

$$
\int_{Q_{0}}|J(x, f)| d x \lesssim \sum_{k=1}^{\infty} \epsilon_{k}<\infty .
$$

By (3.12) there exists a constant $\beta$ such that

$$
K(x) \leq \frac{\beta}{\epsilon_{k}}, \quad x \in 2 Q \backslash Q, Q \in \mathcal{Q}_{k} .
$$

We now define the numbers $\epsilon_{k}$ explicitly by setting

$$
\epsilon_{k}=\frac{\beta}{\Psi^{-1}(k)}
$$

Because $\int_{1}^{\infty} \frac{\Psi^{\prime}(s)}{s} d s<\infty$, the change of variables

$$
s=\Psi^{-1}(t)
$$

shows that

$$
\int_{1}^{\infty} \frac{d t}{\Psi^{-1}(t)}<\infty
$$


Thus the integral criterion for convergence of series establishes (3.8). By (3.12) and (3.14),

$$
\exp \Psi(K(x)) \leq \exp \Psi\left(\frac{\beta}{\epsilon_{k}}\right)=\exp k, \quad x \in 2 Q \backslash Q, Q \in \mathcal{Q}_{k},
$$

and thus

$$
\int_{Q_{0}} \exp \Psi(K(x)) d x \leq C \sum_{k=1}^{\infty}\left(2^{-n} e\right)^{k}<\infty .
$$

Next we will show that $f$ maps a set of measure zero to a set of positive measure by showing that

$$
Q_{0} \subset f\left(\bigcap_{k=1}^{\infty} \bigcup_{Q \in \mathcal{Q}_{k}} Q\right)
$$

recall that the Cantor set $\bigcap_{k=1}^{\infty} \bigcup_{Q \in \mathcal{Q}_{k}} Q$ has measure zero. From the construction it follows that for each $k=1,2,3, \ldots$

$$
f_{k}\left(\bigcup_{Q \in \mathcal{Q}_{k}} Q\right) \subset f_{k}\left(\bigcup_{Q \in \mathcal{Q}_{k+1}} 2 Q\right) \subset f_{k+1}\left(\bigcup_{Q \in \mathcal{Q}_{k+1}} Q\right)
$$

Since $Q_{0} \subset f_{1}\left(\cup_{Q \in \mathcal{Q}_{1}} Q\right)$, denoting

$$
H_{k}=\bigcup_{Q \in \mathcal{Q}_{k}} Q
$$

we have $Q_{0} \subset f_{k}\left(H_{k}\right) \subset f_{l}\left(H_{k}\right)$ for all $l \geq k \geq 1$. Now $\left(H_{k}\right)$ is a decreasing sequence of compact sets, whence

$$
Q_{0} \subset \bigcap_{k=1}^{\infty} \bigcap_{l \geq k} f_{l}\left(H_{k}\right) \subset \bigcap_{k=1}^{\infty} f\left(H_{k}\right) \subset f\left(\bigcap_{k=1}^{\infty} H_{k}\right) .
$$

Notice that $f$ is not open: it follows from the construction that $f\left(\partial Q_{0}\right)=$ $\partial Q_{0} \subset f\left(\right.$ int $\left.Q_{0}\right)$ whence $f\left(Q_{0}\right)=f\left(\right.$ int $\left.Q_{0}\right)$. Because $f\left(Q_{0}\right)$ is a nonempty compact set, $f\left(\operatorname{int} Q_{0}\right)$ is not open. To prove non-discreteness of $f$, let

$$
G_{k}=\bigcup_{l \geq k} f\left(\bigcup_{Q \in \mathcal{Q}_{l}} \operatorname{int} 2 Q \backslash Q\right) .
$$

Then the sets $G_{k}$ are dense and open, and by the Baire category theorem their intersection is nonempty. But if $y \in \cap_{k} G_{k}$, then $f^{-1}(y)$ is an infinite compact set and thus it is not discrete.

Remark 3.1. The example can be easily modified to show sharpness of the condition $(\Phi-1)$ by setting

$$
\epsilon_{k}=2^{-k n} \Phi\left(2^{k}\right)
$$

in place of (3.14). 


\section{Acknowledgements}

J. K., P. K., J. O. and X. Z. are supported in part by the Academy of Finland, project 39788, and J. K. also by the foundation Vilho, Yrjö ja Kalle Väisälän rahasto. J. M. is supported by the Research Project CEZ J13/98113200007, Grant No. 201/00/0767 of Czech Grant Agency (GA ČR) and Grant No. 165/99 of Charles University (GA UK).

\section{References}

[1] Astala, K., Imaniec, T., Koskela, P. and Martin, G.: Mappings of BMO-bounded distortion. Math. Ann. 317 (2000), no. 4, 703-726.

[2] David, G.: Solutions de l'equation de Beltrami avec $\|\mu\|_{\infty}=1$. Ann. Acad. Sci. Fenn. Ser. A I, Math. 13 (1988), no. 1, 25-70.

[3] Giannetti, F., Inaniec, T., Onninen, J. and Verde, A.: Estimates of Jacobians by subdeterminants. J. Geom. Anal. 12 (2002), no. 2, 223-254.

[4] Greco, L.: Sharp integrability of nonnegative Jacobians. Rend. Mat. Appl. (7) 18 (1998), no. 3, 585-600.

[5] Iwaniec, T., Koskela P. And Martin, G.: Mappings of BMOdistortion and Beltrami type operators. J. Anal. Math. 88 (2002), 337-381.

[6] Iwaniec, T., Koskela P. And Onninen, J.: Mappings of finite distortion: Monotonicity and continuity. Invent. Math. 144 (2001), no. 3, $507-531$.

[7] Iwaniec, T. and Martin, G.: The Beltrami equation. Mem. Amer. Math. Soc., to appear.

[8] Iwaniec, T. and Martin, G.: Geometric function theory and non-linear analysis. Oxford Mathematical Monographs. The Clarendon Press, Oxford University Press, 2001.

[9] Kauhanen, J., Koskela, P. and Malý, J.: Mappings of finite distortion: Discreteness and openness. Arch. Ration. Mech. Anal. 160 (2001), no. $2,135-151$.

[10] Kauhanen, J., Koskela, P. and Malý, J.: Mappings of finite distortion: Condition N. Michigan Math. J. 49 (2001), no. 1, 169-181.

[11] Koskela, P. And Zhong, X.: Minimal assumptions for the integrability of the Jacobian. Manuscript.

[12] Manfredi, J. And Villamor, E.: An extension of Reshetnyak's theorem. Indiana Univ. Math. J. 47 (1998), no. 3, 1131-1145.

[13] Reshetnyak, Yu. G.: Space Mappings with Bounded Distortion. Sibirsk. Mat. Z. 8 (1967), 629-658. 
[14] Reshetnyak, Yu. G.: Space Mappings with Bounded Distortion. Trans. of Mathematical Monographs 73. American Mathematical Society, 1989.

[15] Rickman, S.: Quasiregular mappings. Ergebnisse der Mathematik und ihrer Grenzgebiete (3) [Results in Mathematics and Related Areas (3)] 26. Springer-Verlag, Berlin, 1993.

Recibido: 11 de mayo de 2001

Janne Kauhanen

Department of Mathematics and Statistics

University of Jyväskylä

P.O. Box 35

FIN-40351 Jyväskylä, Finland

jpkau@maths.jyu.fi

Pekka Koskela

Department of Mathematics and Statistics

University of Jyväskylä

P.O. Box 35

FIN-40351 Jyväskylä, Finland pkoskela@maths.jyu.fi

Jan Malý

Department KMA of the Faculty of Mathematics and Physics

Charles University

Sokolovská 83

CZ-18675 Praha 8, Czech Republic

maly@karlin.mff.cuni.cz

Jani Onninen

Department of Mathematics and Statistics

University of Jyväskylä

P.O. Box 35

FIN-40351 Jyväskylä, Finland jaonnine@maths.jyu.fi

Xiao Zhong

Department of Mathematics and Statistics

University of Jyväskylä

P.O. Box 35

FIN-40351 Jyväskylä, Finland zhong@maths . jyu .fi 